\title{
An Improved Pair-wise Exchange Heuristic for the Dynamic Plant Layout Problem
}

\author{
Jaydeep Balakrishnan $^{1}$ \\ Chun Hung Cheng ${ }^{2}$ \\ Daniel G. Conway ${ }^{3}$
}

Revised version published in International Journal of Production Research, 38, 13, 2000, 30673077.

1. Faculty of Management, University of Calgary, Calgary, Alberta T2N 1N4, Canada

2. Department of Systems Engineering and Engineering Management, The Chinese University of Hong Kong, Shatin, Hong Kong. Ph: +852 26098322 Email: chcheng@ se.cuhk.hk

3. Department of Decision and Information Sciences, Graduate School of Business, University of Florida, FL 32611 - 7169, USA Ph: (352) 3929599 Email: conwaydg@ufl.edu

Address for Correspondence:

\author{
Jaydeep Balakrishnan \\ Faculty of Management \\ University of Calgary \\ Calgary Alberta T2N 1N4 CANADA \\ Ph: (403) $2207844 \quad$ Fax: (403) 2820095 \\ Email: balakris@ucalgary.ca
}




\title{
An Improved Pair-wise Exchange Heuristic for the Dynamic Plant Layout Problem
}

\begin{abstract}
Much of the research in facility layout has focused on static layouts where the material handling flow is assumed to be constant during the planning horizon. But in today's market-based, dynamic environment, layout rearrangement may be required during the planning horizon to maintain layout effectiveness. A few algorithms have been proposed to solve this problem. They include dynamic programming and pair-wise exchange. In this paper we propose an improved dynamic pair-wise exchange heuristic based on a previous method published in this journal. Tests show that the proposed method is effective and efficient.
\end{abstract}

Keywords: Dynamic facility layout, heuristics, pair-wise exchange, dynamic programming 


\section{An Improved Pair-wise Exchange Heuristic for the Dynamic Plant Layout Problem}

\subsection{Introduction}

This paper investigates the design of facility layouts based on a multi-period planning horizon. During this horizon, the material handling flows between the different departments in the layout may change. This necessitates a more sophisticated approach than the much-researched static plant layout problem (SPLP) approach. The dynamic plant layout problem (DPLP) extends the SPLP by assuming that the material handling flows can change over time. This in turn might necessitate layout rearrangement during the planning horizon.

In an environment where flows do not change over a long period of time, the SPLP is justified. But in today's market based and dynamic environment, such flows can change quickly. Page (6) reports that on average, $40 \%$ of a company's sales come from new products. In the dynamic environment, we can still use the static approach in two ways. The first is to use a short planning horizon so that during this horizon the flows are fairly constant. The disadvantage is that after the short horizon, if the relative flows change, the layout will have to be rearranged ad hoc. Otherwise the result will be an inefficient layout. Rearranging layouts frequently without prior planning can result in operational disruptions and excess rearrangement costs. The second approach is to use a long planning horizon and disregard the changes in flow. There will be no rearrangement costs, but this may result in the layout being inefficient throughout the horizon.

The dynamic approach to layout corrects the above deficiency. In the dynamic approach, layouts are planned based on a multi-period time horizon. During this time if the material flow changes warrant it, one or more layout rearrangements may be planned for. The importance of good layout planning can be gauged from the fact that annually over $\$ 250$ billion is spent in the U.S. alone on layouts that require planning and re-planning $(8, \mathrm{p} 5)$. 


\subsection{Literature review:}

The first major research on the DPLP was undertaken by Rosenblatt (7). He uses dynamic programming (DP) to solve the DPLP. The trade-off here is between the flow (material handling) cost within the facility versus the shifting costs for the departments that might need to be relocated within the facility. Let

$\mathrm{L}_{\mathrm{i}} \quad$ Layout $\mathrm{i}$

$\mathrm{A}_{\mathrm{ij}} \quad$ Rearrangement (sum of department shifting costs) cost when changing from layout $\mathrm{L}_{\mathrm{i}}$ to layout $\mathrm{L}_{\mathrm{j}}$. This cost is independent of the period in which it occurs.

$\mathrm{F}_{\mathrm{it}} \quad$ Material handling cost for layout $\mathrm{L}_{\mathrm{i}}$ in period t. This is obtained from the SPLP solution.

$\mathrm{C}^{*}{ }_{\text {it }} \quad$ Minimum Total Costs (material handling and shifting) for all periods up to $t$ where $\mathrm{L}_{\mathrm{i}}$ is used in period $\mathrm{t}$.

$\mathrm{S}_{\mathrm{it}} \quad$ Sum of all shifting costs for all periods up to $\mathrm{t}$ associated with $\mathrm{C}^{*}{ }_{\mathrm{it}}$.

The combination of layouts with the minimum total cost is chosen based on the following recursive relationship:

$$
\mathrm{C}^{*}{ }_{\mathrm{it}}=\operatorname{Min}_{\mathrm{i}}\left\{\mathrm{C}_{\mathrm{j}(\mathrm{t}-1)}^{*}+\mathrm{A}_{\mathrm{ij}}\right\}+\mathrm{F}_{\mathrm{jt}}^{*}
$$

The DP is solved using backward recursion. Each period in the planning horizon forms a stage. It can be shown that $(N !)^{t}$ options would have to be explicitly or implicitly evaluated in order to find the optimal solution, where $N$ is the number of departments in the layout and $t$ is the number of periods in the planning horizon. Even for a six department, five period problem, $(N !)^{t}$ is $1.93 * 10^{14}$ combinations, a very 
large number indeed! Thus obtaining optimal solutions for large problems is not possible. Rosenblatt suggests a heuristic solution using a few good (or even random) static layouts to make the problem tractable. These good layouts could be generated using a SPLP heuristic such as CRAFT. So a problem with $(n !)^{t}$ options where $n \ll N$, can be solved. However, now there is no guarantee of optimality for the DPLP.

Urban (9) proposes a steepest descent pair-wise exchange heuristic for the dynamic layout problem. This procedure combines the static and dynamic layout problem into one unlike DP where the DPLP is separated into static and dynamic phases. The results indicate that it performs better than DP and only slightly worse than optimal. The computational requirements are also quite low for this method whereas for dynamic programming it could be substantial.

The pair-wise exchange procedure is similar to CRAFT (1). The difference is that shifting costs are included. This heuristic makes use of forecast windows, $m$, to find different sets of good layout plans for the planning horizon. The forecast window is the number of periods being considered when the pair-wise exchange is performed. It ranges from one to the number of periods $t$. Using an initial layout and pair-wise exchanges, one set of layouts is obtained for the given planning horizon in each forecast window.

For example, when the forecast window is 1 , i.e., $m=1$, in each application of the pairwise exchange, only material flows from one period are considered. This is seen in Figure 1, where a five period planning horizon is assumed. An assumed or existing initial layout is used to find the most appropriate layout for period 1 by pair-wise exchanges considering the material flows in period 1 only. Then this newly generated appropriate layout for period 1 is used as the initial layout for period 2. Pairwise exchange is now used to determine a good layout for period 2 by considering the material flows in period 2 only. This process is repeated until the end of the planning horizon. Thus a layout plan for the entire planning horizon is obtained. 


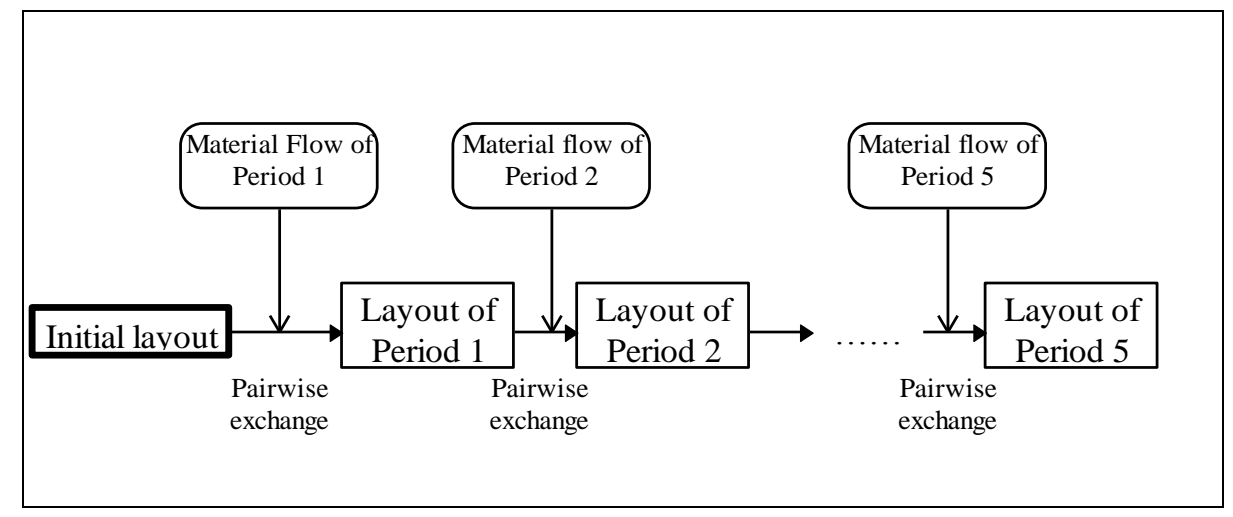

Figure 1: Pair-wise exchange when forecast window $m=1$

When the forecast window is equal to 2 , the material flows in period 1 and period 2 are added to determine the layout in period 1 using pair-wise exchange. Similarly, flow costs in periods 2 and 3 are combined in determining the layout in period 2 . Thus a look-ahead principle is employed. For every $m$, a layout plan can be obtained. However one modification that is needed for some windows is shown in Figure 2, where $m=4$. Data from period 1 through 4 are used to determine a layout for period 1 , and data from period 2 through 5 are used for the layout in period 2. For the layout in period 3, data from periods 3 through 6 would be needed. Since period 6 does not exist, only data from periods 3 through 5 are used in determining the layout for period 3. For period 4, only data from periods 4 and 5 are used. Finally in period 5, only the data from period 5 is used. The forecast window principle can also be seen in Figure 3. 


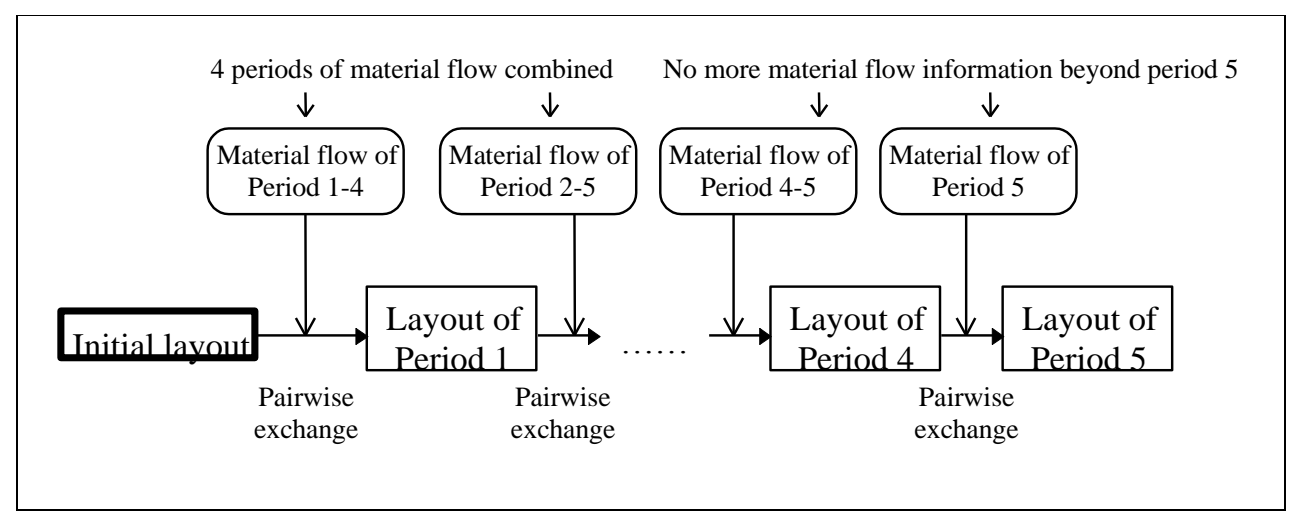

Figure 2: Pair-wise exchange when forecast window $m=4$

For each period (from 1 to $t$ in sequence), the exchange of locations of any pair of departments will change the total flow cost in that period. In addition, the rearrangement cost of shifting from the previous period's layout may change. In pair-wise exchange, an $N$ department layout has ${ }_{N} C_{2}$ number of possible pairs. After considering all ${ }_{N} C_{2}$ pair-wise exchanges, the pair of departments that results in the greatest reduction in the sum of the costs of material flow and rearrangement will be switched in location to generate the new layout. The pair-wise exchange continues until no further improvement can be found. The total cost of the layout plan is the sum of the costs of rearrangement and material flow in all the periods. Since each forecast window gives a layout plan, the plan with the lowest cost is selected as the final solution.

Urban's method provides good solutions and is computational efficient. Thus it is a very practical approach for the DPLP. Some other methods are also available to solve the DPLP. A comprehensive review of these is available in Balakrishnan and Cheng(2). For recent reviews of the static layout problem see Meller and Gau(5), and Kusiak and Heragu(4). 


\subsection{The Proposed Heuristic Methods}

Two improvements are proposed for Urban's heuristic. The first one involves working backward from the final solution provided by Urban's heuristic. The second involves combining Urban's method with DP.

\section{1 Backward method of pair-wise exchange heuristic:}

Urban's heuristic is forward pass in nature. Once a layout for a period is determined, it is never changed in subsequent periods. Thus the quality of the solution in a later period layout depends on the quality of the preceding layouts. This is one disadvantage of the procedure. The backward pass approach reduces this disadvantage. In the backward method, initially Urban's heuristic is used to solve the DPLP. This results in a solution for each forecast window, $m$, as shown in Figure 3. Then a backward pass pairwise exchange is performed on each of these solutions as is also shown in Figure 3 , resulting in $m$ backward pass solutions. The best of these is selected as the final solution. The exchange starts in period $(t-1)$ and goes backward until the first period of the planning horizon. In a five period problem, the backward pass begins with the layout in period 4 (Figure 4). Pair-wise exchange procedures are performed on this layout and if an improvement is found, the appropriate switch is made in that period. Then the procedure is repeated for period 3. The backward pass procedure ends when period 1 is evaluated. Figure 4 also illustrates one major difference between the backward pass and the forward pass methods. In the forward pass only the rearrangement costs between period $(t-1)$ and $t$ are considered, as the layout for period $(t+1)$ is not yet known. In backward pass the rearrangement costs between periods $(t-1)$ and $t$, and the rearrangement costs between periods $t$ and $(t+1)$ are considered since a completed layout plan is available from the forward pass. Another difference is that only material flows in the relevant forward pass period is used regardless of the relevant forward pass forecast window. Material flows from different periods are never added in performing the pair-wise exchange. In other words, the 
forecast window is always equal to one in the backward pass method.

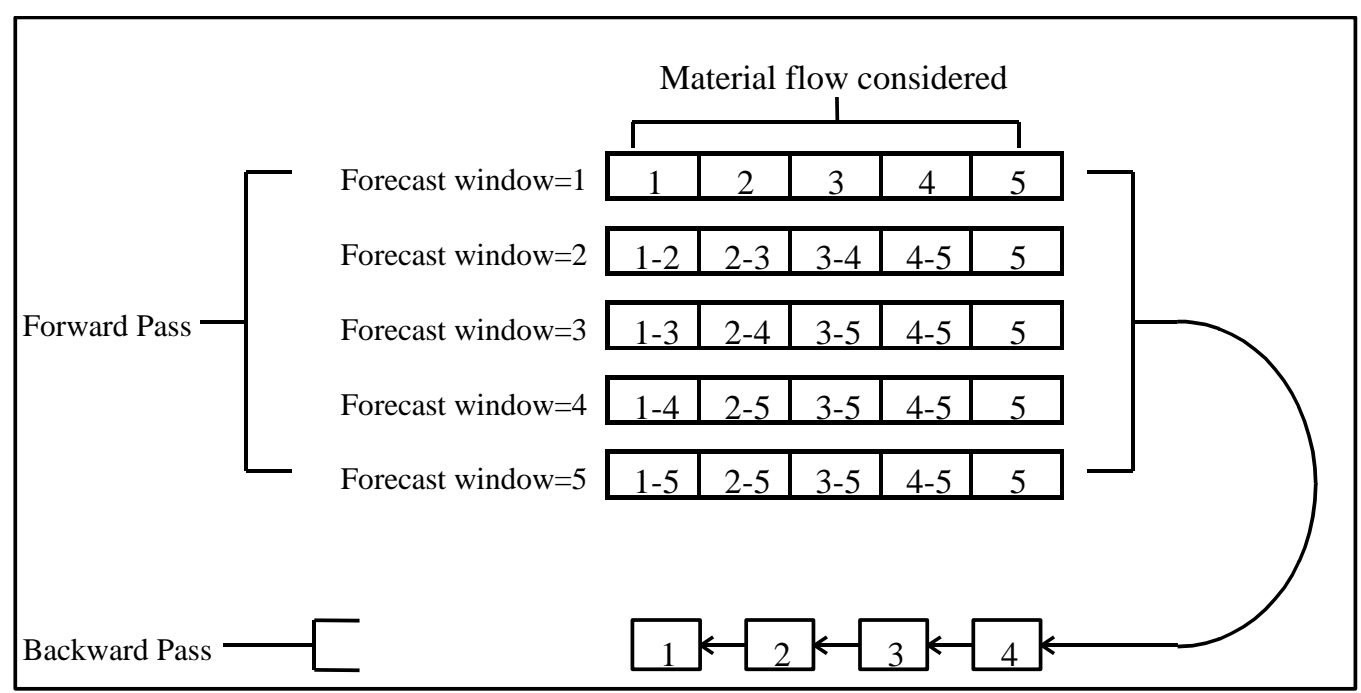

Figure 3: Backward Pass

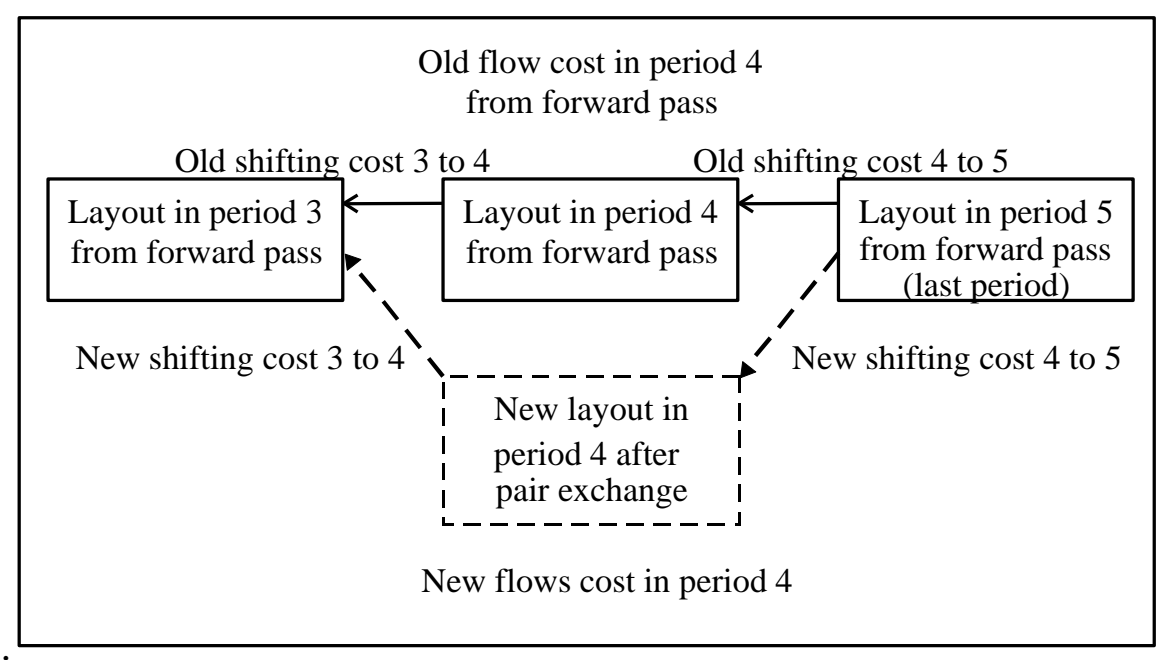

Figure 4: Backward exchange for period 4

Since the backward pass is performed on each layout plan generated from the forward pass, the backward approach will never generate a layout that is worse then the forward pass. If costs cannot be reduced, no exchange will take place in the backward pass. 


\subsection{Combining Urban's heuristic and Dynamic Programming:}

In Urban's pair-wise exchange heuristic, each forecast window gives a layout plan. Within each plan there may be up to $t$ different layouts, if each period requires a different layout. Moreover, there are $m$ different forecast windows and since $m=t$, there could be up to $t \times m$ or $t^{2}$ different layouts generated by Urban's procedure. These layouts can then be used as the states in Rosenblatt's DP procedure that was explained earlier. This is shown in Figure 5.

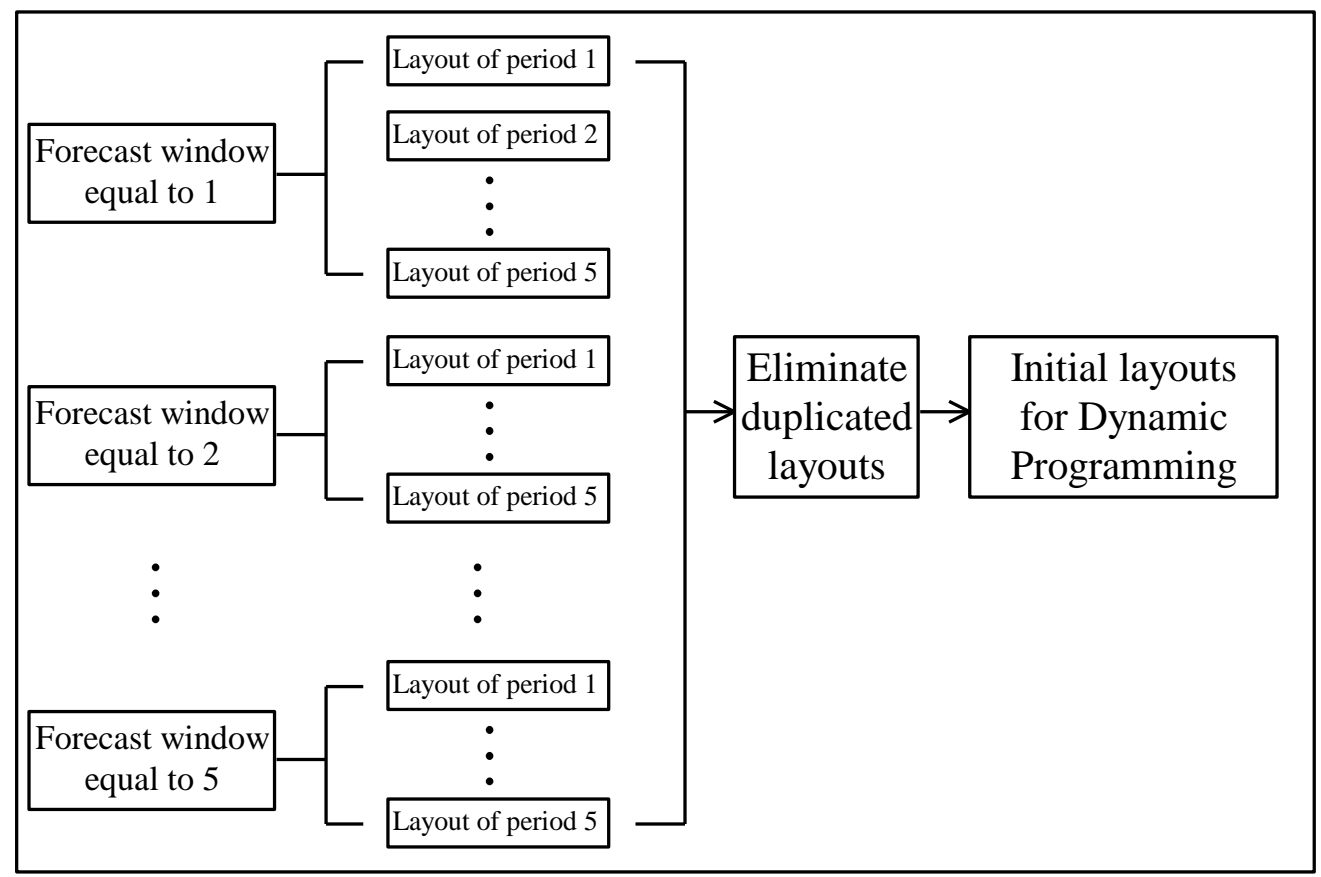

Figure 5: Combined pair-wise and DP approach

This method is appealing because the layouts generated by Urban's pair-wise exchange tend to be good. In addition, the number of layouts (states) in the DP resulting from this method will be reasonable as $t$ is rarely greater than ten. Thus this DP can easily be solved in order to improve the pair-wise exchange result. Moreover as in the backward pass, since the forward pass solution is embedded in the DP, it will give a layout plan as least as good as the layout plan generated by the pair-wise exchange. 


\subsection{Test Setup}

The proposed improvements were tested using problems that consisted of six, fifteen and thirty departments. The shapes of these are explained in the appendix. In each of these three categories, five and ten period planning horizons were considered. This resulted in $3 \times 2$ or 6 different settings. The method used to generate these problems is similar to that in Balakrishnan et al. (3). In each setting eight different problems or data sets were used.

In the six department problems, all 6 ! or 720 possibilities in each period were evaluated using $\mathrm{DP}$, thus giving us the optimal solution. As a result the performance of the pair-wise exchange heuristic and the proposed improvements could be compared to the optimal solution. In the fifteen and thirty department problems, 15 ! and 30 ! proved to large to generate all the possibilities. Thus Rosenblatt's suggestion, of using good static layouts generated by CRAFT as states in the DP procedure, was implemented. Seventy two different good layouts were generated by CRAFT in each period using random initial layouts resulting in $72 \times 5$ or 360 states in the five period problems and 720 states in the ten period problems for the DP method. Randomly generated layouts were also used as states.

Since Urban's pair-wise exchange heuristic is sensitive to the initial layouts used, different initial layouts had to be generated. Two methods were used to generate the initial layouts. The first method generated these randomly. The second method used CRAFT to come up with good initial layouts. In the six and fifteen department problems, seventy two CRAFT solutions were generated in each period using random initial layouts. These were used as initial layouts for pair-wise exchange. This resulted in $72 \times 5$ or 360 initial CRAFT based layouts for the five period problems and 720 initial CRAFT based layouts for the ten period problems. However, for the 30 department problems, solving 360 or 720 pair-wise exchange problems for each of the eight data sets would have been very time consuming. Thus only ten 
initial layouts for the five period problems ( 2 per period $\times 5$ periods) and twenty initial layouts for the ten period problems were used to generate pair-wise exchange solutions.

\subsection{Computational Results}

The detailed results are shown in the appendix. There are six tables, one for each setting. Each table for the fifteen and thirty department problems gives the minimal cost solution for each of the eight problems sets (Data 1 to Data 8) using the following methods:

1 Pair-wise exchange heuristic using initial layouts generated with CRAFT and then combined with DP (COMBINE CRAFT).

2 Pair-wise exchange heuristic using initial layouts generated with CRAFT and then subjected to the backward pass algorithm (BACKWARD CRAFT).

3 Pair-wise exchange heuristic using initial layouts generated with CRAFT (HEUR CRAFT).

4 Pair-wise exchange heuristic using initial layouts generated randomly (HEUR RANDOM).

5 Dynamic programming using static layouts generated with CRAFT (DYN CRAFT).

6 Dynamic programming using static layouts generated randomly (DYN RANDOM).

The first two are the improved methods suggested in this paper. The third is a modification to Urban's procedure proposed by us. The fourth is basically Urban's procedure, except that more initial layouts are used. The last two are Rosenblatt's methods. In each of tables A3 through A6, a $B$ in a cell implies that it gave the best solution of all the methods for that data set.

In the six department problems, in the DP all the 720 possible layouts were included. Similarly for pair-wise exchange, 720 possible initial layouts were used. Thus the number of different procedures reduced to four; dynamic programming (DYN), Urban's pair-wise exchange (HEUR), pairwise exchange combined with DP (COMBINE), and pair-wise exchange combined with backward pass (BACKWARD). In tables A1 and A2, a $B$ in a cell implies that it gave the best solution among HEUR, 
COMBINE and BACKWARD. DYN naturally always gave the optimal solution. The results are summarised in Table 1 below.

Table 1: The summary results

\begin{tabular}{|c|c|c|c|c|c|c|}
\hline $\begin{array}{l}\text { Dept. } \\
\text { Size }\end{array}$ & $\begin{array}{l}\text { Planning } \\
\text { Horizon }\end{array}$ & $\begin{array}{l}\text { Average } \% \\
\text { above best } 1\end{array}$ & $\begin{array}{l}\text { Minimum \% } \\
\text { above best }\end{array}$ & $\begin{array}{c}\text { Maximum } \% \\
\text { above best }\end{array}$ & \multicolumn{2}{|c|}{$\begin{array}{c}\text { Average \% } \\
\text { above optimal } 4\end{array}$} \\
\hline \multirow{4}{*}{6} & \multirow[b]{2}{*}{5} & & & & Improved & $0.03^{\mathrm{a}}$ \\
\hline & & & & & Original & $0.45^{\mathrm{b}}$ \\
\hline & \multirow[b]{2}{*}{10} & & & & Improved & $0.13^{\mathrm{a}}$ \\
\hline & & & & & Original & $1.38^{\mathrm{b}}$ \\
\hline \multirow[b]{2}{*}{15} & 5 & 0.2 & 0.0 & 0.55 & & \\
\hline & 10 & 0.25 & 0.05 & 0.54 & & \\
\hline \multirow[b]{2}{*}{30} & 5 & 0.28 & 0.0 & 0.68 & & \\
\hline & 10 & 0.39 & 0.15 & 0.68 & & \\
\hline
\end{tabular}

1 The best solution in every case was provided by COMBINE CRAFT or BACKWARD CRAFT. These percentages represent the average difference (from the eight data sets) between results provided by COMBINE CRAFT or BACKWARD CRAFT (whichever was better) and HEUR CRAFT and HEUR RANDOM (whichever was better). DYN CRAFT and DYN RANDOM are not included in this table as these are not part of the primary basis for comparison. They performed the worst in all cases.

2 These values represent the minimum difference in the eight data sets between results provided by COMBINE CRAFT or BACKWARD CRAFT (whichever was better) and HEUR CRAFT and HEUR RANDOM (whichever was better). A value of 0.0 indicates that in at least one of the data sets, the results were identical.

3 These values represent the maximum difference in the eight data sets between results provided by COMBINE CRAFT or BACKWARD CRAFT (whichever was better) and HEUR CRAFT and HEUR RANDOM (whichever was better).

4 Optimal solutions were available only in the six department problems.

a These values represent the average difference in the eight data sets between the optimal solution and the results provided by COMBINE or BACKWARD (whichever was better).

$\mathrm{b}$ These values represent the average difference in the eight data sets between the optimal solution and the results provided by HEUR CRAFT and HEUR RANDOM (whichever was better).

The results show that the proposed modifications to Urban's algorithm resulted in improved results in each and every one of the fifteen or thirty department problems except one, i.e. thirty one of thirty two. In that one case there was no improvement. In addition, the results in Table 1 show a 
tendency toward better improvements in the larger problems, i.e., when the number of departments is thirty and/or when number of periods is ten. In the six department problems, the proposed improvements resulted in the optimal solution in six and three data sets out of the eight respectively for the five and ten department problems. The corresponding numbers without the improvements is three and none. As a result the average deviation from optimality is greater for Urban's original heuristic (even with the additional initial layouts).

In the fifteen department problems, the COMBINE CRAFT method appears to work better than the BACKWARD CRAFT method. However in the six and thirty department problems, the same cannot be said. The best solutions seem to be more evenly distributed between COMBINE CRAFT and BACKWARD CRAFT.

There also appears to be no advantage in using CRAFT based initial solutions for Urban's pairwise exchange heuristic. Only in ten of the thirty two problems did it improve the solution. This appears to be a consistent result in both the fifteen and thirty department problems. Consistent with the result in Urban's paper, DYN CRAFT and DYN RANDOM perfomed the worst. In addition consistent with the results found in Balakrishnan et al., DYN CRAFT performs better than DYN RANDOM.

Since the DPLP is a design problem, decisions are not required very frequently. This coupled with the fact that the suggested improvements are based on pair-wise exchange and solving small DPs, computation time was not a factor in this study.

\subsection{Conclusion}

Though the DPLP is more relevant than the SPLP in the present day business operating environment, relatively little research has been done in it. Among the few methods proposed for this problem, Urban's pair-wise exchange technique is a practical approach. It is not difficult to implement as it is based on the CRAFT approach. Therefore it can also be used to solve large problems. In this paper 
we have suggested some computationally efficient improvements to Urban's pair-wise exchange procedure. Tests show that these improvements are worth implementing. In almost every case it does improve on the results provided by Urban's procedure. Finally the study also confirms some previous results regarding the DPLP. 


\section{References}

1. Armour G.C., and Buffa E.S., "A heuristic algorithm and simulation approach to relative allocation of facilities", Management Science, 9, 2, 294-300, 1963.

2. Balakrishnan J. and Cheng C.H., "Dynamic layout algorithms: a state-of-the-art survey", $O M E G A$, 26,4, 507-521, 1998.

3. Balakrishnan J., Jacobs R.F., Venkataramanan M.A., "Solutions for the constrained dynamic facility layout problem”, European Journal of Operations Research, 57, 280-286, 1992.

4. Kusiak A., and Heragu S.S., "The facility layout problem", European Journal of Operational Research, 29, 229-251, 1987.

5. Meller R.D. and Gau K.Y., "The facility layout problem: recent and emerging trends and perpectives", Journal of Manufacturing Systems, 15, 5, 351-366, 1996.

6. Page A.L., "PDMA new product development survey: performance, and best practices", Paper presented at PDMA Conference, Chicago, Nov. 13, 1991.

7. Rosenblatt M.J., “The dynamics of plant layout”, Management Science, 32, 1, 1986, 76-86.

8. Tompkins J.A, White J.A., Bozer Y.A., Frazelle E.H., Tanchoco J.M.A. and Trevino J., Facilities Planning, New York: Wiley, 1996.

9. Urban T.L., "A heuristic for the dynamic facility layout problem”, IIE Transactions, 25, 4, 57-63, 1993. 


\section{APPENDIX}

\section{Layout Shapes (Rows by Columns)}

6 departments: 2 by 3

15 departments: 3 by 5

30 departments: 5 by 6

\section{Results}

Table A1: 6 departments, 5 periods

All 6! = 720 layouts used.

\begin{tabular}{|l|l|l|l|l|l|l|l|l|}
\hline METHOD & Data 1 & Data 2 & Data 3 & Data 4 & Data 5 & Data 6 & Data 7 & Data 8 \\
\hline DYN & 106419 & 104834 & 104320 & 106399 & 105628 & 103985 & 106439 & 103771 \\
\hline HEUR & 106419 B & 105262 & 104791 & 107243 & 105628 B & 104810 & 106439 B & 105005 \\
\hline COMBINE & 106419 B & 104834 B & 104320 B & 106583 B & 105628 B & 104053 B & 106439 B & 105005 \\
\hline BACKWARD & 106419 B & 104834 B & 104791 & 107243 & 105628 B & 104053 B & 106439 B & 103771 B \\
\hline
\end{tabular}

$\underline{\text { Table A21: } 6 \text { departments, } 10 \text { periods }}$

All 6! = 720 layouts used.

\begin{tabular}{|l|l|l|l|l|l|l|l|l|}
\hline METHOD & Data 1 & Data 2 & Data 3 & Data 4 & Data 5 & Data 6 & Data 7 & Data 8 \\
\hline DYN & 214313 & 212134 & 207987 & 212530 & 210906 & 209932 & 214252 & 212588 \\
\hline HEUR & 215283 & 212877 & 209952 & 213845 & 219944 & 213516 & 216769 & 215818 \\
\hline COMBINE & 214313 B & 212138 B & 207987 B & 213185 B & 210906 B & 210360 B & 215165 B & 213967 \\
\hline BACKWARD & 215143 & 212293 & 209817 & 213706 & 210944 & 212584 & 216593 & 212823 B \\
\hline
\end{tabular}

\section{Table A3: 15 departments, 5 periods}

CRAFT: $\quad 72$ layouts improved by CRAFT in each period, total 360 layouts

Random: $\quad 72$ random layouts in each period, total 360 layouts

\begin{tabular}{|l|l|l|l|l|l|l|l|l|}
\hline METHOD & Data 1 & Data 2 & Data 3 & Data 4 & Data 5 & Data 6 & Data 7 & Data 8 \\
\hline COMBINE CRAFT & 485139 & 485809 B & 491363 B & 486955 & 489987 B & 489606 B & 489625 & 492244 B \\
\hline BACKWARD CRAFT & 484142 B & 485809 B & 491381 & 484625 B & 490494 & 490086 & 489345 B & 492644 \\
\hline HEUR CRAFT & 485139 & 485809 B & 492015 & 487313 & 491899 & 490146 & 489897 & 492644 \\
\hline HEUR RANDOM & 485139 & 485809 B & 492015 & 487313 & 491899 & 490146 & 489897 & 493758 \\
\hline DYN CRAFT & 486276 & 490013 & 495343 & 489338 & 494605 & 492877 & 492417 & 495515 \\
\hline DYN RANDOM & 508496 & 510624 & 519488 & 512838 & 512834 & 512377 & 510582 & 519629 \\
\hline
\end{tabular}

$\underline{\text { Table A4: } 15 \text { departments, } 10 \text { periods }}$ 
CRAFT: $\quad 72$ layouts improved by CRAFT in each period, total 720 layouts

Random: $\quad 72$ random layouts in each period, total 720 layouts

\begin{tabular}{|l|l|l|l|l|l|l|l|l|}
\hline METHOD & Data 1 & Data 2 & Data 3 & Data 4 & Data 5 & Data 6 & Data 7 & Data 8 \\
\hline COMBINE CRAFT & 989486 B & 984307 B & 988363 B & 977318 B & 984894 B & 974989 B & 988521 & 989293 \\
\hline BACKWARD CRAFT & 991092 & 985863 & 992601 & 977765 & 986731 & 975598 & 988244 B & 989154 B \\
\hline HEUR CRAFT & 991155 & 986338 & 993886 & 982606 & 987985 & 975658 & 990102 & 989693 \\
\hline HEUR RANDOM & 991929 & 986338 & 993471 & 982606 & 987985 & 977271 & 989712 & 990548 \\
\hline DYN CRAFT & 992274 & 987397 & 995889 & 984308 & 990347 & 981629 & 991016 & 992987 \\
\hline DYN RANDOM & 1038203 & 1029594 & 1045949 & 1025717 & 1029920 & 1024417 & 1038686 & 1045932 \\
\hline
\end{tabular}

\section{Table A5: 30 departments, 5 periods}

CRAFT: $\quad 2$ layouts improved by CRAFT in each period, total 10 layouts

Random: $\quad 2$ random layouts in each period, total 10 layouts

\begin{tabular}{|l|l|l|l|l|l|l|l|l|}
\hline METHOD & Data 1 & Data 2 & Data 3 & Data 4 & Data 5 & Data 6 & Data 7 & Data 8 \\
\hline COMBINE CRAFT & 581014 & 576671 & 580850 & 573611 B & 559662 B & 567463 B & 567917 B & 577488 B \\
\hline BACKWARD CRAFT & 579742 B & 572505 B & 579712 B & 574967 & 560064 & 568094 & 567917 B & 577636 \\
\hline HEUR RANDOM & 580225 & 576390 & 581997 & 576040 & 562085 & 568657 & 568676 & 578905 \\
\hline HEUR CRAFT & 581014 & 576671 & 582206 & 575245 & 561577 & 569133 & 567917 & 579774 \\
\hline
\end{tabular}

CRAFT: $\quad 72$ layouts improved by CRAFT in each period, total 360 layouts

Random: $\quad 72$ random layouts in each period, total 360 layouts

\begin{tabular}{|l|l|l|l|l|l|l|l|l|}
\hline METHOD & Data 1 & Data 2 & Data 3 & Data 4 & Data 5 & Data 6 & Data 7 & Data 8 \\
\hline DYN CRAFT & 594590 & 585349 & 593992 & 580370 & 566628 & 574727 & 576602 & 581241 \\
\hline DYN RANDOM & 611794 & 611873 & 611664 & 611766 & 603327 & 608151 & 607134 & 618287 \\
\hline
\end{tabular}

Table A6: 30 departments, 10 periods

CRAFT: $\quad 2$ layouts improved by CRAFT in each period, total 20 layouts

Random: $\quad 2$ random layouts in each period, total 20 layouts

\begin{tabular}{|l|l|l|l|l|l|l|l|l|}
\hline METHOD & Data 1 & Data 2 & Data 3 & Data 4 & Data 5 & Data 6 & Data 7 & Data 8 \\
\hline COMBINE CRAFT & 1170945 & 1174542 & 1170476 & $\begin{array}{l}1155828 \\
\text { B }\end{array}$ & $\begin{array}{l}1131484 \\
\text { B }\end{array}$ & $\begin{array}{l}1148184 \\
\text { B }\end{array}$ & $\begin{array}{l}1145916 \\
\text { B }\end{array}$ & $\begin{array}{l}1171856 \\
\text { B }\end{array}$ \\
\hline BACKWARD CRAFT & $\begin{array}{l}1169139 \\
\mathrm{~B}\end{array}$ & $\begin{array}{l}1173759 \\
\mathrm{~B}\end{array}$ & $\begin{array}{l}1169478 \\
\mathrm{~B}\end{array}$ & 1157124 & 1135303 & 1152107 & 1154961 & 1172538 \\
\hline HEUR RANDOM & 1172070 & 1177458 & 1171729 & 1159742 & 1139455 & 1150567 & 1153666 & 1179190 \\
\hline HEUR CRAFT & 1170945 & 1178261 & 1171816 & 1158903 & 1139080 & 1153336 & 1155770 & 1179290 \\
\hline
\end{tabular}

CRAFT: $\quad 72$ layouts improved by CRAFT in each period, total 720 layouts

Random: $\quad 72$ random layouts in each period, total 720 layouts

\begin{tabular}{|l|l|l|l|l|l|l|l|l|}
\hline METHOD & Data 1 & Data 2 & Data 3 & Data 4 & Data 5 & Data 6 & Data 7 & Data 8 \\
\hline DYN CRAFT & 1195988 & 1202683 & 1198235 & 1169999 & 1143509 & 1159383 & 1158983 & 1179200 \\
\hline DYN RANDOM & 1228411 & 1231978 & 1231829 & 1227413 & 1209014 & 1221986 & 1212273 & 1242347 \\
\hline
\end{tabular}

